\title{
Design and Application of Desktop Cloud Experimental Teaching Platform
}

\author{
Yang Sheng \\ School of Management \\ Wuhan University of Science and Technology \\ Wuhan 430065, China
}

\begin{abstract}
In order to reform the teaching and management traditional laboratories, and propel the informatization and intelligentization of experimental teaching, this paper constructs a new experimental teaching platform based on virtualization technology and cloud computing technology, for addressing the disadvantages of traditional experimental teaching platform. The new platform adopts Citrix XenServer for server virtualization, and builds an experimental teaching environment with Citrix XenDesktop. It involves various links of experimental teaching, such as experimental teaching arrangement, student experiment attendance and management, experimental equipment and network monitoring, etc. A convenient mobile laboratory is built by using mobile terminals such as mobile phones and tablets. With the online learning function, students can choose courses to study independently. The application of desktop cloud experimental teaching platform greatly improves the efficiency of laboratory management, realizes the dynamic management of experimental teaching process, and improves students' independent learning ability and innovative practice quality.
\end{abstract}

Keywords-Desktop Cloud; virtualization; experimental teaching platform; laboratory management

\section{INTRODUCTION}

In the information era, neither theoretical teaching nor practical teaching in college and universities can proceed without computer technologies. However, the current construction and management of laboratories still face many problems, impeding the development of education, especially the practical teaching [1]. It is urgent for colleges and universities to build an intelligent, efficient and integrated information laboratory with emerging technologies.

Cloud computing is a computing technology that has been continuously developed and promoted in recent years. As a typical application of cloud computing, desktop cloud, through virtualization application, can release uniformly managed teaching cloud resources to various operating terminals and provide services in the form of virtual desktop [2]. The desktop cloud model has obvious advantages over the traditional model. Desktop cloud is more stable, easier to operate and maintain, more economical, and can effectively address the traditional university laboratory deficiencies. Desktop cloud is constantly moving towards practical application, providing new ideas and approaches for the construction of university laboratories [3-4].

\section{MANAGEMENT DRAWBACKS OF TRADITIONAL LABORATORIES IN COLLEGES AND UNIVERSITIES}

\section{A. Labs are not always available}

It is difficult to improve the comprehensive quality of students and cultivate their innovative consciousness and practical ability in the laboratory under the traditional closed management mode [5]. Because of the lack of perfect information laboratory management system in traditional laboratories, the opening time, space and content of them are limited. Students can only complete experiments in accordance with instructions of the teachers of professional experimental courses, and they are not able to choose the practical courses they are interested in or propose self-developed projects for research, which hinders their improvement of innovation ability. In addition, the schedule of some practical courses is tight, and students can only rush to accomplish the experiment within a limited class time and they cannot make full use of the experimental resources in their extracurricular time, which can seldom achieve good experimental results.

\section{B. Lack of trace management in experimental teaching}

Traditional laboratories still rely on manual management and use paper files to record the arrangement, progress and classroom situation of experimental courses. However, the experimental instructor can hardly know each student's computer operation period and the completion of experimental tasks only through paper records. Due to the lack of collection of teaching data, it is difficult for the experimental instructor to obtain students' feedback information, so there might be a deviation in the curriculum syllabus. At the same time, laboratory managers are unable to carry out effective inspection and supervision of experimental courses. The statistical works of computer operation time, laboratory equipment and software have some problems such as inaccurate data, which cannot provide powerful data to support the laboratory management decision.

\section{Lack of effective monitoring of laboratory equipment}

With the continuous improvement of the status of practical teaching in higher education, the number of experimental courses and laboratory equipment has increased. Under the manual management mode of laboratory, many problems arise, such as the inconvenience of equipment search and statistics, unclear condition of equipment (such as in use, suspension or 
scrap), and no accountability for equipment damage. Once the network or equipment fails, lab managers need to spend a lot of time in troubleshooting, affecting the order of experimental teaching and teaching quality.

\section{DesKTOP Cloud TeChNOLOGY}

The National Institute of Standards and Technology (NIST) defines cloud computing as a model for enabling ubiquitous, convenient, on-demand network access to a shared pool of configurable computing resources (e.g., networks, servers, storage, applications, and services) that can be rapidly provisioned and released with minimal management effort or service provider interaction [6].

Cloud computing is composed of three service models, that is, IaaS (Cloud Infrastructure as a Service), PaaS (Cloud Platform as a Service) and SaaS (Cloud Software as a Service) [7]. According to the provider, it can be divided into a private cloud and public cloud [8].

Desktop cloud, namely desktop virtualization, represents one of the important applications of cloud computing. Desktop cloud is a combination of PaaS and SaaS. Users can access the virtual personal desktop system and various applications on the server side with the help of special client programs or browsers through thin client devices or any other devices connected to the network. The thin client device of the desktop cloud is equipped with an independent embedded operating system, and accesses the virtual desktop of cloud server through remote desktop protocol. All the resource consumption supporting the operation of the operating system and application software occurs on the cloud server side. The cloud terminal does not undertake the tasks of computing and storage, whose main task is to promote human-computer interaction [9].

At present, desktop virtualization is divided into two main technical frameworks: VDI (Virtual Desktop Infrastructure) and VOI (Virtual OS Infrastructure) [10]. Abiding by "integrated management, centralized computing, distributed display”, VDI virtual desktop architecture centralizes all client operations on the server of the data center for processing, and the client is only responsible for input and output and interface display [11]. VDI establishes a virtual machine pool in the background through virtualization technology, integrates the management of the entire desktop image, compresses the video frames of remote display results and transmits them to the client for restoring display. Therefore, the client in VDI mode does not need to configure any operating system and applications. However, VDI mode requires hardware emulation and thin protocol, so it has higher demands on the network. Without adding media acceleration function, HD video, Adobe Flash, IP (VoIP) and other computing or graphics intensive applications are not suitable for VDI mode. Virtual desktop architecture of VOI realizes standardization and instant distribution from desktop application delivery to OS. VOI mode adopts the principle of "centralized management and distributed computing”. Virtual system realizes data redirection of physical storage media directly in I/O layer, and takes over computer hardware platform from the boot stage. In VOI mode, desktop operating system and application software are uniformly deployed on the server side, and then the operating system and application software are distributed on demand to the client side in the form of data stream, and calculated on the client side [12]. Because VOI makes full use of the local computing resources of the terminal, it has low dependence on the network and server. Even if the network and server fail, it can also use the cache to run the virtual desktop normally. VOI mode also enjoys strong image-intensive computing ability and good peripheral compatibility.

\section{LAB DESIGN SCHEME BASED ON DESKTOP CLOUD TECHNOLOGY}

\section{A. The overall architecture of desktop cloud}

In the current desktop cloud technology solutions, VDI virtual desktop architecture is the mainstream architecture and deployment mode. The desktop cloud platform based on VDI mode is composed of terminal access layer, desktop session management layer, virtualization platform layer and storage layer. The division of work for each layer is shown in Fig. 1.

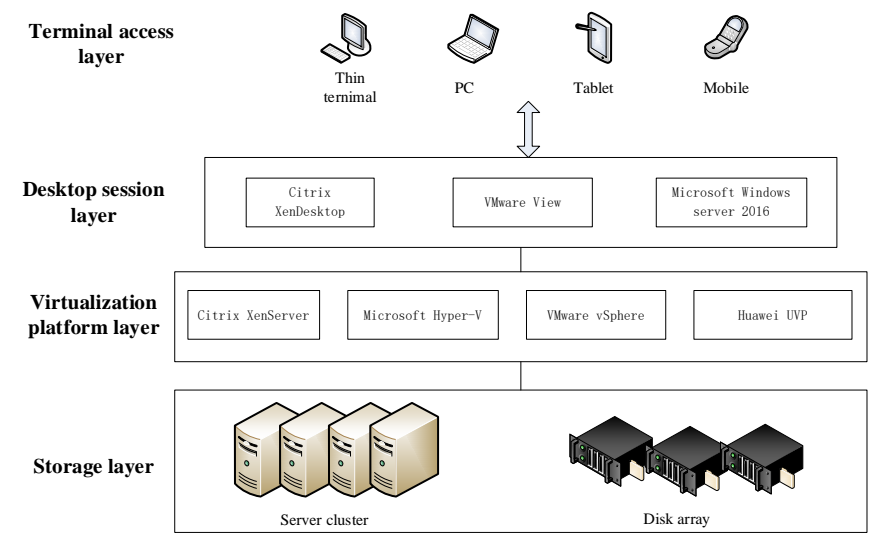

Fig. 1. Composition of Desktop Cloud

(1) Terminal access layer: specific terminal devices, such as thin client, PC, mobile terminal, etc.; co-access the server virtual desktop through RDP, ICA, PCoIP and other remote desktops.

(2) Desktop session management layer: responsible for user authentication, authorization and desktop allocation when accessing the session; session maintenance, state and monitoring after the establishment of the session. Common products include: Citrix XenDesktop, VMware View, Microsoft Windows Server 2016, etc.

(3) Virtualization platform layer: by installing virtualization platform software on the server, the server is pooled, and the virtual machine resource pool is provided for the upper virtual machine to use and schedule resources. Nowadays, the mainstream virtualization platform software involves Citrix XenServer, Microsoft Hyper-V, VMware vSphere, Huawei UVP and so on.

(4) Storage layer: storage resource pool of desktop cloud platform consists of server cluster and disk array. Storage layer provides system space, data space and space needed by desktop cloud management system for the virtual desktop [13]. The application software and the operating system are installed on 
the server, and then the application software and the operating system are delivered to the client terminal safely as services through virtualization platform software.

\section{B. Implementation scheme of desktop cloud laboratory}

At present, the mainstream desktop virtualization protocols mainly include: HDX (Citrix), PCoIP (Vmware), RDP (Microsoft RemoteFX), SPICE (Redhat), etc. By comparing various virtual desktop protocols (see Table 1), the desktop cloud platform of Citrix XenDesktop is chosen.

TABLE I. COMPARISON OF MAINSTREAM DESKTOP VIRTUALIZATION PROTOCOLS

\begin{tabular}{c|ccccc}
\hline Protocols & $\begin{array}{c}\text { Transmission } \\
\text { bandwidth }\end{array}$ & Image display & Binary audio & Video playback & Transport security \\
\hline HDX & low & high & high & high & high \\
PCoIP & high & high & low & low & Citrix \\
RDP & high & low & middle & middle & VMware \\
SPICE & middle & middle & high & middle & Microsoft \\
\hline
\end{tabular}

The cloud platform of the school laboratory is set as 4 computer laboratories with 215 terminals in total. The operating system is Windows 7 (64 bits). The terminal is configured as YX-218 terminal equipment $(\mathrm{CPU}>1.8 \mathrm{GHz}$,

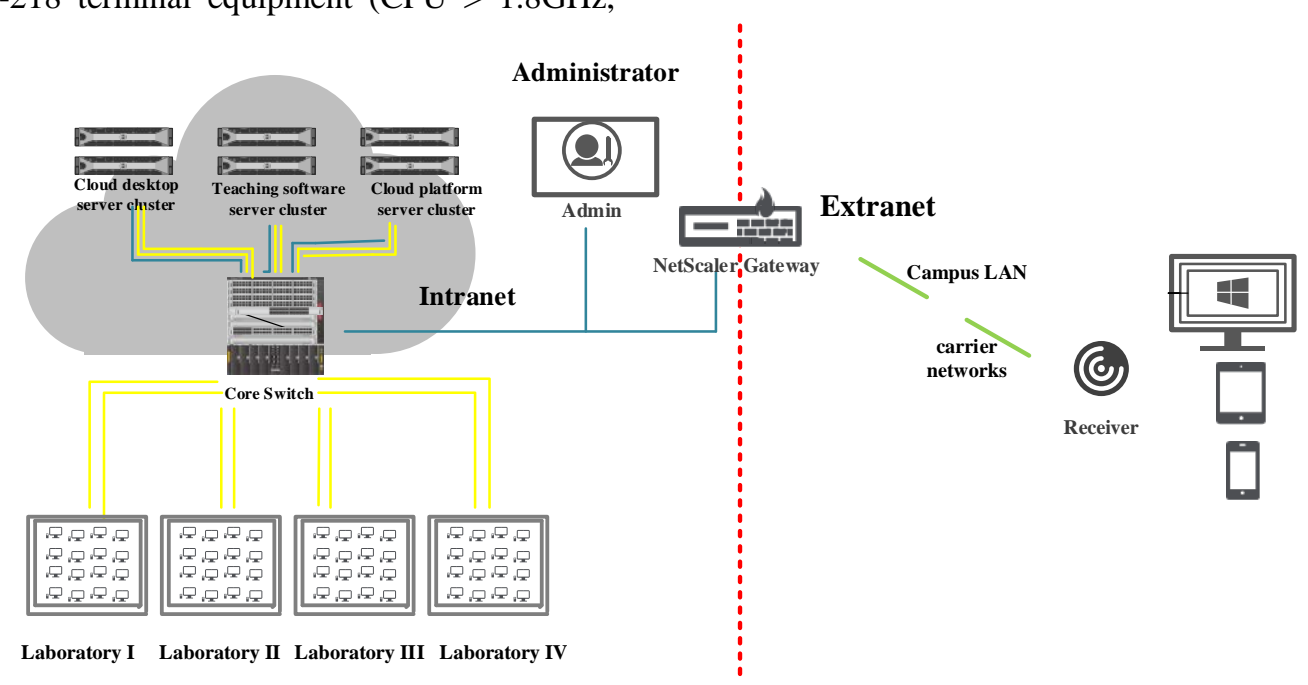
three H3Cs, two Dells and four HPs. The topology of the desktop cloud laboratory is shown in Fig. 2.

Fig. 2. Topology of desktop cloud lab

\section{The functional design of desktop cloud experimental teaching platform}

The functions of desktop cloud experimental teaching platform [14] mainly include management of experiment appointment, such as teachers' announcement of courses, students' online course selection, and laboratory managers' review and appointment; experiment attendance management, such as student registration, experimental attendance, experiment record query, etc.; equipment and network management, such as check of network and equipment operation status, fault warning, etc.; lab resource management, such as e-tutorials, e-courseware, online courses, exercise testing, etc.; experimental teaching information database, such as mining big data of experimental teaching to generate experimental teaching data report. The most important issue of this platform is to introduce support for mobile terminals such as mobile phones and tablets, which greatly facilitates users. The overall functional structure of the experimental teaching platform is shown in Fig. 3. 


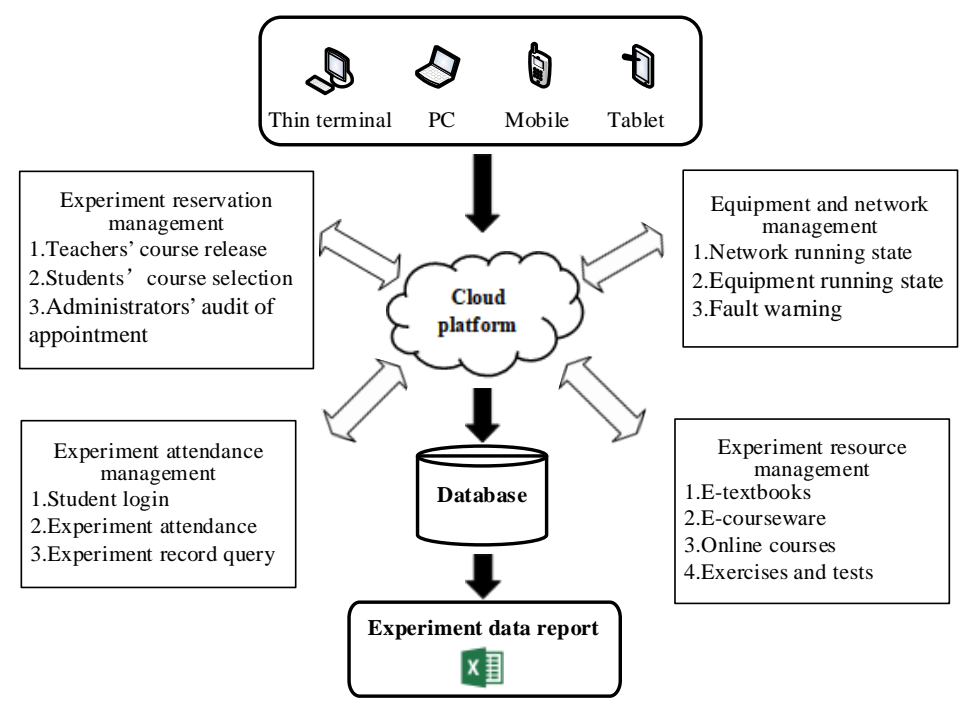

Fig. 3. Functional structure of experimental teaching platform

\section{1) Experiment reservation management module}

This module includes three aspects: students' course selection, teachers' course release and administrators' audit of appointment. Experiment reservation is more flexible. The teacher announces the content and time of the experimental project according to the teaching plan, then students make an appointment according to their own course arrangement, and finally, the teacher reviews the application of students' appointment.

\section{2) Experiment attendance management module}

This module contains login, attendance and query. When students log in the lab software through the lab computer or their own mobile terminal, the attendance system starts to record the time and software used by students if the reservation record exists.

\section{3) Experiment resource management module}

This module builds an online learning platform, which is mainly used for teachers to add teaching resources online, including e-textbooks, e-courseware, online courses, exercises and tests, etc. Online, students can choose courses according to their own needs after logging into the system; Offline, teachers can choose some knowledge to explain to students according to the teaching needs. Meanwhile, teachers can also set tasks for students to learn independently after class to complete assigned exercises, which can assist teaching and realize flipped classroom.

\section{4) Equipment and network management module}

The module mainly includes network running state, equipment running state, fault warning and alarm. By virtue of cloud platform and equipment network management system, an intelligent and unified management platform is built to monitor and manage the running state of the campus network, host, virtual machine and application software 24 hours a day. Lab managers quickly and intuitively monitor and manage all lab equipment and application systems. When the fault occurs, the system can realize fault warning and alarm, inform the laboratory management personnel of the system situation at any time through email and SMS, and point out the fault equipment.

\section{5) Experimental teaching information database}

Using the big data mining function of cloud technology, we can extract useful data from the laboratory system, including the statistics of computer time, the use of laboratory software and equipment, the progress of students' experiments, etc., and automatically generate the reports or statements required by the college. Through statistical analysis of experimental teaching information data, it provides support for teaching management and teaching reform.

\section{6) Mobile Laboratory}

Using cloud desktop technology, we can achieve experimental information sharing through a standard web browser to provide application services. Students can use the software on the laboratory cloud platform on their own computers, mobile phones, tablets and other mobile terminals to further improve the openness of the laboratory.

\section{CONCLUSIONS}

The era of cloud computing has arrived. Desktop cloud technology laboratory boasts of great advantages in software and hardware costs, as well as in the maintenance, management and upgrading of the laboratory in the later stage. In this paper, Citrix XenServer is used to virtualize the server, and Citrix XenDesktop is used to create the experimental teaching environment. From the technical and laboratory management level, it is feasible and effective to build the desktop cloud technology laboratory. Currently, desktop cloud technology laboratory runs steadily, which optimizes the experimental teaching process and experimental management process and saves the cost of experimental manpower and resources, hence greatly improving the efficiency of experimental teaching. 


\section{REFERENCES}

[1] Zhang Wuqi, Wang Xingyu, Sha Yi. Exploration on the Reform of Laboratory Integrated Management System in Colleges and Universities, 2018, 37(11):156-159.

[2] Huang Chenxi, Lin Yongqin. Research and Implementation on Cloud Computing-based Virtual Computer Laboratory [J]. Research and Exploration in Laboratory, 2010, 29(11):178-181.

[3] Cui Guanxun. Design and Implementation of a Computer Experimental Teaching Platform Based on Cloud Computing [J]. Research and Exploration in Laboratory, 2013, 32(10):447-450.

[4] Lu Minrong. On Experimental Teaching Platform Based on "Internet +" Virtual Technology [J]. Computer Applications and Software, 2017(10):135-141.

[5] Chen Xiaoqiao, Sui Zhucui, Zhou Liqing. Strengthening the Construction of Open Experimental Platform to Improve College Students' Independent Innovation Ability [J]. Experimental Technology and Management, 2016, 33(7):1-3.

[6] Jiang Guozhong. On Cloud Computing Technology [J]. Internet of Things Technologies, 2013(12):50-51.

[7] Wang Yu. Research on Computer Course Experiment Teaching Platform Based on Cloud Technology [J]. Journal of Changchun University of Science and Technology (Natural Science Edition), 2017, 40(6):147-150.
[8] Chen Donglin, Fu Min, Chen Ling. Study on Construction Mode of Experimental Teaching Platform in Colleges and Universities Based on Hybrid Cloud [J]. Experimental Technology and Management, 2013, 30(5):63-66

[9] Qin Feng, Huang Shanbin, Qin Weiling. Research on Management of Multimedia Computer Laboratory Based on Desktop Clouds [J] Experimental Technology and Management, 2015, 32(3):164-166.

[10] Huang Jingan. Research and Implementation of Desktop Cloud Architecture in College Teaching Environment [J]. Computer Technology and Development, 2013(12):222-225.

[11] Zhang Kai, Yang Zaiming, Song Huining. Desktop Virtualization Technology in the Multimedia Classroom Management Application [J]. Experimental Technology and Management, 2017, 34(6):131-134.

[12] Zhang Ying, Ling Shiyong. Design and Implementation of Desktop Cloud by Combining VDI with VOI in Multimedia Classroom[J]. Research and Exploration in Laboratory, 2017, 36(10):145-148.

[13] Wang Fan, Chen Chunlian. Construction and Management of Computer Laboratory Based on Desktop Cloud [J]. Experiment Science and Technology, 2015, 13(3):190-193

[14] Wu Xu, Chen ren'an, Wei Dezhi. Design and Application of Laboratory Integrated Management System under Cloud Computing [J]. Research and Exploration in Laboratory, 2015, 34(10): 226-229. 